\title{
Laparoscopic Distal Pancreatectomy: Feasibility Study of Radiofrequency-Assisted Transection in a Porcine Model
}

\author{
Dimitri Dorcaratto, MD, ${ }^{1,2}$ Fernando Burdío, MD, $\mathrm{PhD}^{1,2}$ Dolors Fondevila, $\mathrm{PhD}{ }^{3}$ \\ Anna Andaluz, DVM, PhD, ${ }^{3}$ Ignasi Poves, MD, PhD, Maria Angeles Martinez, MD, \\ Rita Quesada, MSc, ${ }^{1,4}$ Enrique Berjano, $\mathrm{PhD}^{5}$ and Luis Grande, MD, $\mathrm{PhD}^{1,2}$
}

\begin{abstract}
Background and Aim: Despite technological improvements in pancreatic surgery, the incidence and morbidity of pancreatic leak after resection of distal pancreas are persistently high in most series. Laparoscopic distal pancreatectomy (LDP) is today the gold standard procedure for benign and certain malignant neoplasms of the pancreatic body and tail in specialized centers. This study evaluated safety and feasibility of a radiofrequency (RF)-assisted transection device in a porcine model of LDP.

Materials and Methods: LDP was performed on 10 pigs (median weight, $39.6 \mathrm{~kg}$ ) using a new device based on an internally cooled RF-assisted electrode (Coolinside ${ }^{\circledR}$, Apeiron Medical, Valencia, Spain). The animals were subjected to daily observation and then sacrificed and necropsied at 4 weeks postoperatively. Primary end points were the development of postoperative pancreatic fistula using the Pancreatic Anastomotic Leak Study Group definition and/or the presence of abdominal amylase-rich fluid collections or abscesses during necropsy and pathological study and/or dye extravasation from the pancreatic remnant duct. Secondary end points were intra- or postoperative complications, surgery, and transection duration.

Results: No clinically relevant postoperative pancreatic fistulas were observed. In one case a grade A postoperative fistula was diagnosed due to amylase drain concentration of more than $6200 \mathrm{IU} / \mathrm{mL}$ on postoperative day 4. Median peritoneal liquid amylase concentration on postoperative day 4 was $2399.0 \mathrm{IU} / \mathrm{L}$ (range, 819.2$7122.0 \mathrm{IU} / \mathrm{L}$ ), similar to the median plasma amylase level of 1520.8 IU/L (range, 1015.3-4056.6 IU/L). Median surgery time was 93.5 minutes (range, 46.0-140.0 minutes), and median transection time was 4.5 minutes (range, 2.0-26.0 minutes). There was one postoperative wound infection. There were no postoperative deaths or major complications. During the histopathological study, the surgical margin of the remaining pancreas showed a common pattern with a central area of necrosis surrounded by granulomatous infiltrate and fibrosis. Ductal obliteration was observed. No purulent inflammatory infiltrate or abscesses were present.

Conclusion: Experimental findings suggest that performing pancreatic transection with Coolinside in a animal model of LDP is feasible and safe.
\end{abstract}

\section{Introduction}

D istal pancreatectomy (DP) with or without spleen preservation has been applied with increasing frequency in the management of a broad range of pancreatic pathologies during the last two decades. ${ }^{1,2}$ Advances in operative technique and perioperative management have led to a substantial decrease of postoperative mortality, achieving rates between $0 \%$ and $6 \%{ }^{3,4}$ However, morbidity rates after this procedure have not changed during the last 20 years, ranging from $10 \%$ to $57 \%$ in larger series. ${ }^{1-4}$ Pancreatic fluid leak from the pancreatic remnant and subsequent fistula formation have been identified as the most frequent and serious post-DP complications. ${ }^{5,6}$

Although laparoscopic DP (LDP) is safe and effective ${ }^{7-9}$ and is considered by some authors the best approach to

${ }^{1}$ General Surgery Department, Hospital del Mar, Barcelona, Spain.

Departments of ${ }^{2}$ Surgery and ${ }^{3}$ Animal Medicine and Surgery, Veterinary Faculty, Universitat Autonoma de Barcelona, Barcelona, Spain.

${ }^{4}$ Department of Biomedical Engineering, Universitat Politecnica de Catalunya, Barcelona, Spain.

${ }^{5}$ Biomedical Synergy, Electronic Engineering Department, Universitat Politecnica de Valencia, Valencia, Spain. 
left-sided pancreatic pathology in need of resection, ${ }^{8}$ no reduction in postoperative pancreatic fistula rates has been demonstrated using this surgical approach in comparison with the conventional open approach. ${ }^{8}$ The difficulty in preventing pancreatic leak after DP has resulted in various methods of closing the pancreatic stump. However, no method has been clearly proven to be superior to others, as reported in a recent meta-analysis. ${ }^{3,10,11}$

Radiofrequency (RF) energy-assisted devices have been used in both experimental and clinical surgery to manage sealing of the pancreatic remnant after $\mathrm{DP}^{10,12}$ with initially encouraging results in reducing postoperative pancreatic fistula.

In 2009 Burdio et al. ${ }^{13}$ reported their results in liver resection using a new RF-assisted device that was used to transect hepatic tissue, to coagulate hepatic vessels up to $4 \mathrm{~mm}$ in diameter, and to seal bile ducts. Our hypothesis is that this RFassisted device, which has previously demonstrated a high capability of closing resected tissues, ${ }^{13-16}$ could be suitable for transecting pancreatic parenchyma and sealing the main and secondary pancreatic ducts. The aim of this study was therefore to evaluate safety and viability of this RF device in a porcine LDP model.

\section{Materials and Methods}

\section{Instruments}

The RF device is a laparoscopic version of the Coolinside ${ }^{\circledR}$ device (Apeiron Medical, Valencia, Spain). Its operating performance has been described in detail elsewhere. ${ }^{15,16}$ In brief, it consists of a handheld instrument that simultaneously conducts the two surgical tasks of coagulation/sealing and cutting. Coagulation and sealing are obtained from a 3-mmdiameter electrode connected to an RF generator (model CC-1, Radionics, Burlington, MA). The electrode is internally cooled with chilled saline solution $\left(0^{\circ} \mathrm{C}\right)$ by means of a peristaltic pump (Radionics). Mechanical cutting is carried out by a sharp blade attached distally to the electrode. The tissue is coagulated by the proximal zone of the electrode with a backward movement and is subsequently transected by the blade, which cuts only the amount of tissue previously coagulated and sealed. ${ }^{15,16}$

\section{Animals}

Ten female Landrace pigs obtained from the farm of the Universidad Autonoma of Barcelona (Barcelona, Spain) were used for the experimental procedures. All aspects of this study were performed as part of an animal research protocol according to the guidelines approved by the Government of Catalonia's Animal Care Committee.

\section{Preoperative care}

Preoperative and anesthesia care was provided by fully trained veterinary staff members. A combination of azaperone and ketamine $(4 \mathrm{mg} / \mathrm{kg}$ i.m. and $10 \mathrm{mg} / \mathrm{kg}$ i.m., respectively) was used for initial sedation. Intravenous access was obtained by a marginal ear vein cannulation using largegauge venous catheters, and intravenous analgesia was given before anesthesia $(0.4 \mathrm{mg} / \mathrm{kg}$ i.m. morphine and $0.2 \mathrm{mg} / \mathrm{kg}$ i.v. meloxicam).

Anesthetic induction was performed with propofol (4 mg/ $\mathrm{kg}$ i.v.) and maintained with a mixture of isoflurane $(1.5 \%-$
$2 \%$ ) and oxygen (100\%) once the animal was endotracheally intubated.

Perioperative fluids consisted of Ringer's solution at $10 \mathrm{~mL} / \mathrm{kg} /$ hour. Oxygen saturation and heart rate were monitored using pulse oximetry placed on the ear.

The animals were secured supine on the operating table, and sterile drapes were applied after a betadine preparation of the abdomen.

\section{Surgical technique}

All LDP procedures were performed by the same surgical team using a four-port laparoscopic approach, following the porcine model pancreatic dissection technique described by Truty et al. ${ }^{10}$ After pancreatic dissection, approximately $4-6 \mathrm{~cm}$ of the distal pancreas was transected using the RF device. The same device was used to seal and coagulate the pancreatic remnant. The main pancreatic duct was neither identified nor sutured after transection of the distal pancreas in order to test the sealing efficacy of the RF device on the pancreatic parenchyma. The resected specimen was then removed through the umbilical orifice. A silicone drain was then placed in the resection bed and brought out of the animal's abdomen through the 5-mm inferior right trocar orifice. The proximal end was subcutaneously tunneled to the animal's back and connected to a reservoir. All wounds were closed in standard fashion. The animals were allowed to awaken from anesthesia and were extubated when clinically indicated.

\section{Postoperative care}

The animals were housed in large individual facilities at our institution. Antibiotics (amoxicillin, $20 \mathrm{mg} / \mathrm{kg}$ i.m., every 24 hours) were administered for the first 3 postoperative days. All animals were given water ad libitum for the first 24 hours and subsequently fed twice daily with pig chow thereafter.

All animals were inspected twice a day for the first 7 postoperative days in order to identify any suspicious clinical sign of pancreatic leak or sepsis and to monitor debit and state of abdominal drains. All animals received buprenorphine (0.02-0.03 mg/kg i.m., every 12 hours) for the first 24 postoperative hours and meloxicam $(0.2 \mathrm{mg} / \mathrm{kg}$ i.m., every 24 hours) for postoperative analgesia on the first 3 postoperative days.

\section{Necropsy}

Four weeks after the initial procedure all animals were again anesthetized, intubated, and ventilated as described above. Exploratory laparotomy was performed, and the peritoneal cavity was assessed for excessive adhesions or any undrained fluid collections/abscess. The pancreatic stump was identified, skeletonized, and photographed (Fig. 1). The remnant pancreas (stump, uncinate process, and head) was dissected, removed, and temporarily placed in $10 \%$ buffered neutral formalin. The main pancreatic duct was identified and cannulated with an angiocatheter. A 1:5 dilution of Black China dye was then injected into the pancreatic duct in a retrograde fashion to assess for macroscopic dye extravasation from the pancreatic stump. Thereafter the specimen was immersed in $10 \%$ buffered neutral formalin for further histopathological processing.

The animals were then sacrificed with a commercial euthanasia solution. 


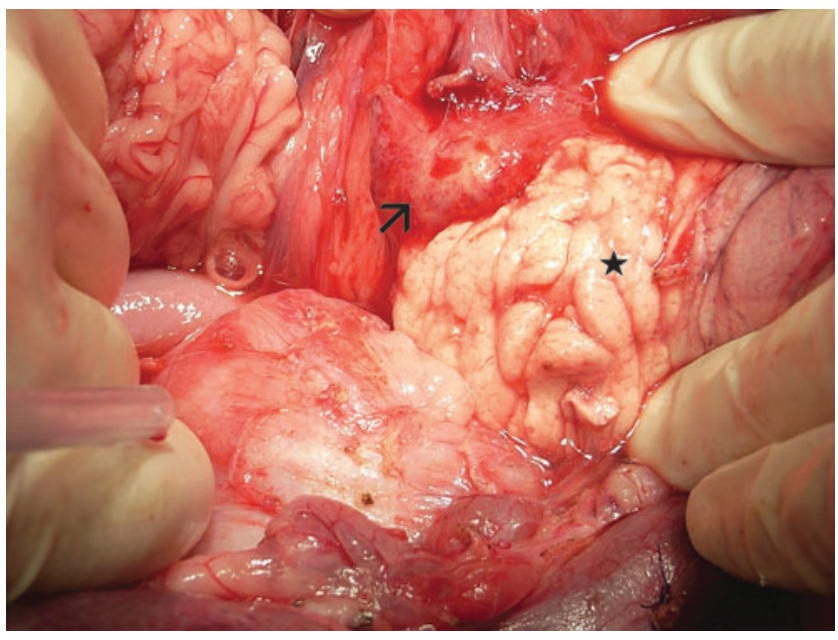

FIG. 1. Macroscopic view of the remaining pancreas 4 weeks after performing laparoscopic distal pancreatectomy. The black star indicates the distal pancreatic remnant. The black arrow highlights fibroid tissue surrounding the transected surface of distal pancreas.

\section{Laboratory determinations}

Serum amylase and glucose levels were obtained before initial incision, 4 hours after intervention, and on postoperative day 4 and 4 weeks postoperatively.

Peripancreatic fluid amylase levels were measured from the drain tube on postoperative day 4 . The drain tube was retired between postoperative days 4 and 7 .

\section{Histopathologic study}

Consecutive sections, $2 \mathrm{~mm}$ thick, were obtained in the margin of the interface of transection, including normal pancreas. Alternate sections were routinely processed, embedded in paraffin, cut at a thickness of $5 \mu \mathrm{m}$, stained with hematoxylin and eosin, and evaluated by light microscopic examination.

\section{Analyzed variables}

The primary outcomes studied in our experiment were the development of postoperative pancreatic leak defined as (1) macroscopic leak (evidence of dye extravasation from the pancreatic stump), (2) any undrained amylase-rich fluid collections/abscess, and (3) greater than threefold drain/serum amylase after the third postoperative day (the definition of a biochemical leak according to the International Study Group on Pancreatic Fistula guidelines ${ }^{6}$ ). Secondary outcomes were operative time, transection time, intraoperative complica- tions, wound infection, and other perioperative clinical parameters (anorexia, emesis, lethargy, and narcotic need).

\section{Statistical analysis}

Data are presented as median and minimum-maximum value. Data collection and analyses were performed with statistical software (SPSS version 16.0, SPSS, Inc., Chicago, IL).

\section{Results}

There were no deviations from the protocol described in the methodology.

\section{Animals}

Median preoperative weight was $39.6 \mathrm{~kg}$ (range, 29.7$74.0 \mathrm{~kg}$ ). All animals increased their body weight during the first postoperative month, with a median pre-necropsy weight of $55.0 \mathrm{~kg}$ (range, $43.0-80.0 \mathrm{~kg}$ ).

\section{Biochemical determinations}

Serum amylase and glucose levels throughout the study are shown in Table 1.

It is of note that at the 4th postoperative hour, all animals showed an increase in serum amylase levels, which returned to levels similar to baseline on postoperative day 4 .

\section{Operative features}

Median operative time was 93.5 minutes (range, 46.0-140.0 minutes), of which a median of 4.5 minutes (range, 2.0-26.0 minutes) was needed for pancreatic transection. No major intraoperative complications were observed. Superficial injuries due to grasper manipulation were observed on gastric wall serosa in 2 cases and repaired laparoscopically with suture on both occasions. These animals showed no clinical or laboratory complications during postoperative follow-up, and macroscopic examination during necropsy showed complete recovery of gastric wall continuity. In 1 case, a superficial lesion of the gastric serosa due to thermal injury during pancreatic transection was observed, which did not affect the subserosal muscular layer. Laparosocopic suture was performed, and the animal did not present any postoperative clinical complication or variation on necropsy findings. No thermal injuries or complications related to the use of the device were otherwise observed. There were no intraoperative deaths.

\section{Postoperative follow-up}

No animals showed lethargy, emesis, anorexia, or supplementary narcotic need during the postoperative period. Only

Table 1. Preoperative and Postoperative (4 Hours, 4 Days, and 4 Weeks Postoperatively) Serum Amylase and Glucose Levels

\begin{tabular}{lcccc}
\hline & & \multicolumn{3}{c}{ Postoperative } \\
\cline { 3 - 5 } & Preoperative & 4 hours & 4 days & 4 weeks \\
\hline Serum amylase (IU/L) & $1396.4(1072.7-3003.8)$ & $2201.9(1179.1-3603.0)$ & $1520.8(1015.3-4056.0)$ & $1356.0(1048.0-2816.1)$ \\
Serum glucose (mg/dL) & $117.4(63.2-210.8)$ & $122.1(85.3-208.4)$ & $115.0(92.6-238.2)$ & $100.8(61.6-187.3)$ \\
\hline
\end{tabular}

Data are median (range) values. 
one trocar orifice infection was observed, which did not require surgical debridement or antibiotic treatment.

\section{Necropsy}

Neither dye extravasation from the pancreatic stump nor any undrained amylase-rich fluid collections or abscesses were observed in gross examination during necropsy. Thus, no clinical or anatomic evidence of pancreatic fistula was observed in any of the animals included in the study.

\section{Amylase drain levels}

Figure 2 shows the animals' amylase concentration in drain fluid on postoperative day 4 . Postoperative measurement was impossible in one animal because of drain loss on postoperative day 1. Median peritoneal liquid amylase concentration on postoperative day 4 was 2399.0 IU/L (range, 819.2$7122.0 \mathrm{IU} / \mathrm{L}$ ). As for biochemical pancreatic leak, using the definition proposed by the International Study Group on Pancreatic Fistula, ${ }^{6}$ a drain fluid amylase level of more than threefold normal serum concentration on postoperative day 4 was only observed in one animal (7122.0 IU/L), which did not present any postoperative clinical complication or free intraabdominal fluid during necropsy (Grade A fistula).

\section{Histopathological study}

The histopathological study of the transection margin of the pancreas stump (Fig. 3) showed a common pattern with a central area of coagulative necrosis surrounded by a granulomatous inflammatory infiltrate, with macrophages and multinucleated giant cells. An intense fibrosis was present, delimiting the necrotic material and the inflammatory infiltrate. The fibrosis reaction completely encircled the main and secundary pancreatic ducts located at the margin of normal pancreas. Scattered infiltrate of lymphocytes, plasma cells, and eosinophils were seen in the fibrous boundary. No purulent inflammatory infiltrate or abscesses were observed in any sample.

\section{Discussion}

The results of this experimental study suggest that performing pancreatic transection with the Coolinside device in an LDP animal model could be feasible and safe.

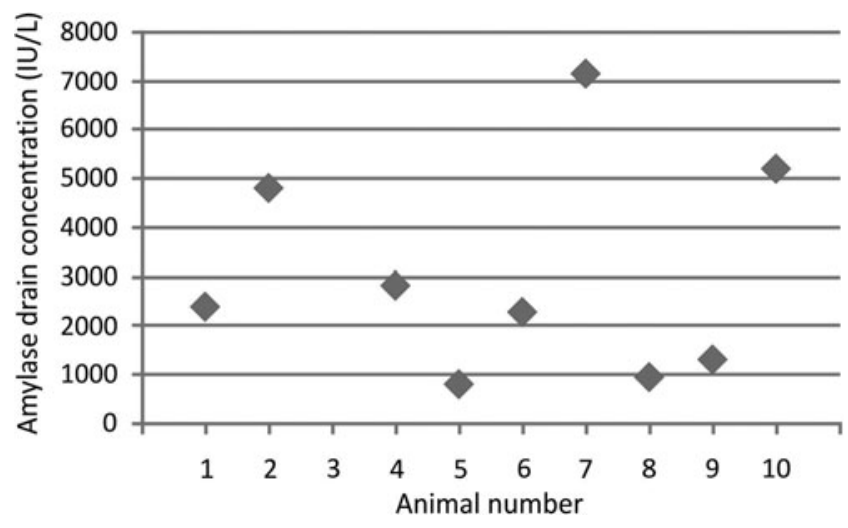

FIG. 2. Drain amylase concentration at postoperative day 4.
Morbidity after DP is still a challenging problem, with postoperative rates ranging from $10 \%$ to $57 \%$ in high-volume centers. ${ }^{1-6,17,18}$ Pancreatic fistula is a main cause of postoperative complications and is associated with numerous further problems, such as intraabdominal abscesses, wound infection, sepsis, electrolyte imbalance, malabsorption, and hemorrhage and with a dramatic increase in healthcare resource utilization. ${ }^{3,17}$

Several surgical resection and closure techniques have been introduced for DP in an attempt to reduce complications, especially pancreatic fistulas. ${ }^{17}$ Unfortunately, none of these has shown a clear superiority ${ }^{3}$ as reported in a recent metaanalysis in which no significant differences were found between stitch suture and stapler closure in relation to pancreatic fistula or intraabdominal abscess rates $(31.2 \%$ and $22.1 \%$ rates of postoperative pancreatic leak, respectively). ${ }^{19}$

In our study, we hypothesized that RF energy could have an obliterative effect on pancreatic ductal structures, as has previously been demonstrated for vascular and biliary structures. ${ }^{16}$ Furthermore, as the RF device used in the present study had previously been proven to be able to seal vessels up to $4 \mathrm{~mm}$ in diameter, ${ }^{16}$ we decided to test its capacity to seal the main pancreatic duct without previous identification and ligation. Our findings on the histopathological examination of the pancreatic remnants 4 weeks after transection confirmed our hypothesis because they showed a common pattern of progressive obliteration of both main and secondary pancreatic ducts (see Fig. 3).

We agree with Truty et al. ${ }^{10}$ that simple main duct ligation and traditional oversewing of the gland with sutures does not completely seal the small pancreatic ducts, leading to persistent extravasation of enzyme-rich pancreatic fluid, and that the porcine model is an excellent approximation to the human pancreas, ${ }^{10}$ with its "soft" texture making it a good model for high-risk pancreatic leak. ${ }^{1,10,20}$ We can speculate that this risk is in part increased by the crushing of the pancreatic tissue and the consequent destruction of small pancreatic ducts due to the pressure applied by a mechanical stapler before cutting and staple application. ${ }^{11}$ In our model no pressure is applied to the pancreatic tissue, and only the previously sealed parenchyma are then cut by the device, thus avoiding the rupture of small pancreatic ducts before sealing.

At gross examination during necropsy, all animals showed the same pattern of minimal postoperative field adhesions, without inflammatory signs, free peritoneal liquids, or abscesses (see Fig. 2). The transection surface of the pancreatic stump presented a thick fibrous surface of more than $1 \mathrm{~mm}$ (see Fig. 3). Under this fibrotic "envelope," a common pattern of sterile coagulative necrosis was found in all specimens, with no signs of necrosis of the pancreatic remnant. No signs of pancreatitis were found on microscopical analysis of the pancreatic remnant, indicating that the coagulative necrosis achieved with RF application does not increase the risk of pancreatic inflammatory reaction, as has been observed by other authors. ${ }^{10}$

This conclusion was confirmed by the absence of clinical signs of pancreatitis and biochemical elevation of serum amylase during postoperative follow-up. No signs were found of the intense pyogranulomatous inflammatory reaction caused by silk suture or of a pattern of peristaple serpentine necrosis due to intermittent crushing of tissue along the staple line, as is caused by mechanical suture. ${ }^{11}$ 

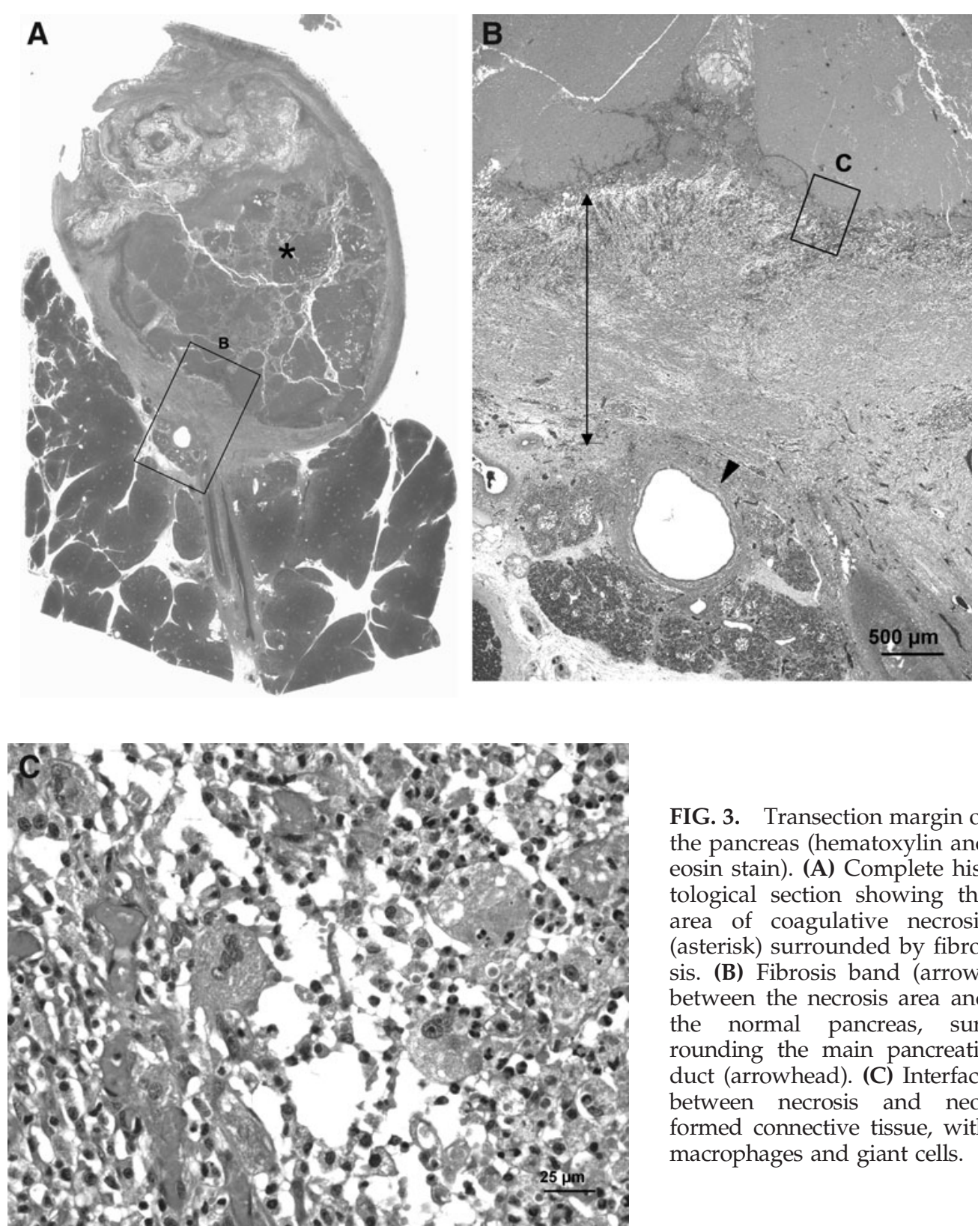

FIG. 3. Transection margin of the pancreas (hematoxylin and eosin stain). (A) Complete histological section showing the area of coagulative necrosis (asterisk) surrounded by fibrosis. (B) Fibrosis band (arrow) between the necrosis area and the normal pancreas, surrounding the main pancreatic duct (arrowhead). (C) Interface between necrosis and neoformed connective tissue, with macrophages and giant cells.

In our opinion the coagulative necrosis of the transected surface caused by thermal injury and the consequent reactive fibrosis could provide a stronger "defense" against pancreatic fluid leak compared with the inflammatory reaction to silk and staplers, and the absence of foreign bodies could decrease the risk of microorganism colonization. A comparative study is needed to confirm our hypothesis.

Another important issue in our study was to evaluate the handling of the Coolinside during LPD. The diffusion of laparoscopic surgery of distal pancreas and the need for simple and ergonomic methods to manage the pancreatic stump during this surgical approach are probably the main reasons for the increasing interest in finding a simple and effective method to cut and seal the pancreatic remnant., $\mathrm{At}$ present, no single instrument adequately addresses simultaneously transection, hemostasis, and sealing of pancreatic ducts. The vast majority of pancreatic parenchymal transections are performed using at least two different instruments, ${ }^{7}$ which involves greater complexity and time-consuming, costly operations.

Our attention was focused on testing the capacity of a single device able to cut and seal the pancreatic tissue without increasing surgical complexity or requiring additional time. Previous comparative experimental studies had shown that the use of the Coolinside device alone for both laparoscopic transection and sealing of liver tissue decreased surgical time and blood loss. ${ }^{16}$

In this study we found no technical difficulty in performing laparoscopic transection with the Coolinside device, as can be seen from the median surgical and resection times. The ability to perform laparoscopic pancreatic transection with no need to identify or suture the main pancreatic duct is certainly the device's main advantage, provided that future studies confirm the low postoperative fistula rates suggested in this feasibility study. The device seems to be very easy to use and permits the sealing of pancreatic ducts and coagulation of 
small pancreatic vessels before the blade cuts the previously coagulated tissue. These preliminary results suggest that this device could provide good hemostasis of both pancreatic tissue and surrounding fat tissue and avoid the need for other coagulating devices during and after pancreatic resection. No intraoperative hemorrhages were observed during our study.

No major complications were found associated with the use of the device, and only one clinically insignificant superficial gastric lesion could be attributed to thermal injury to nearby structures.

\section{Study limitations}

Our study has certain limitations, which should be pointed out. The most important is related to the sample size, which was too small to confirm the efficacy of the Coolinside device to prevent postoperative pancreatic leak. In spite of this limitation, the absence of clinical and macroscopic pancreatic leak is encouraging (only one animal presented a biochemical Grade A pancreatic fistula on the grading scheme of the International Study Group on Pancreatic Fistula).

Certain anatomic differences between pigs and humans limit the model's applicability to pancreatic surgery. ${ }^{11}$ First, the tail of the pancreas is far more intraperitoneal in pigs than in humans, making LDP significantly less challenging in the pig. Second, as most researchers do, ${ }^{11}$ we used as surgical models young pigs, which have little intra- and peripancreatic fat, which could limit the applicability of our results to humans. On the other hand, as stated before, the possibility of performing pancreatic transection and sealing using only one device without the need for main duct identification and ligation seems to make further research on its feasibility for use on humans worthwhile.

\section{Conclusions}

Preliminary results suggest that performing pancreatic transection and sealing with the Coolinside device during LDP is feasible and safe. Based on our results, comparative studies and human trials are needed to assess the clinical utility of this device.

\section{Acknowledgments}

This work received financial support from the Spanish "Plan Nacional de I+D+I del Ministerio de Ciencia e Innovación," grant number TEC2008-01369/TEC, and from the Generalitat Valenciana (Ayudas Complementarias de I+D para Grupos de Calidad ACOMP/2010/008).

\section{Disclosure Statement}

F.B., E.B., and R.Q. declare an interest (stock ownerships) in Apeiron Medical S.L., a company that has a license for the patent application US 2010/137856 A1, on which the device tested in this study is based. D.D., D.F., A.A., M.M., I.P., and L.G. have no conflict of interests or financial ties to disclose.

\section{References}

1. Nathan H, Cameron JL, Goodwin CR, Seth AK, Edil BH, Wolfgang CL, Pawlik TM, Schulick RD, Choti MA. Risk factors for pancreatic leak after distal pancreatectomy. Ann Surg 2009;250:277-281.

2. Balcom JH, Rattner DW, Warshaw AL, Chang Y, Fernandezdel Castillo C. Ten-year experience with 733 pancreatic resections: Changing indications, older patients, and decreasing length of hospitalization. Arch Surg 2001;136: 391-398.

3. Knaebel HP, Diener MK, Wente MN, Buchler MW, Seiler CM. Systematic review and meta-analysis of technique for closure of the pancreatic remnant after distal pancreatectomy. Br J Surg 2005;92:539-546.

4. Goh BK, Tan YM, Chung YF, Cheow PC, Ong HS, Chan WH, Chow PK, Soo KC, Wong WK, Ooi LL. Critical appraisal of 232 consecutive distal pancreatectomies with emphasis on risk factors, outcome, and management of the postoperative pancreatic fistula: A 21-year experience at a single institution. Arch Surg 2008;143:956-965.

5. Shrikhande SV, D'Souza MA. Pancreatic fistula after pancreatectomy: Evolving definitions, preventive strategies and modern management. World J Gastroenterol 2008;14:57895796.

6. Bassi C, Dervenis C, Butturini G, Fingerhut A, Yeo C, Izbicki J, Neoptolemos J, Sarr M, Traverso W, Buchler M. Postoperative pancreatic fistula: An international study group (ISGPF) definition. Surgery 2005;138:8-13.

7. Kooby DA, Chu CK. Laparoscopic management of pancreatic malignancies. Surg Clin North Am 2010;90:427-446.

8. Kooby DA, Gillespie T, Bentrem D, Nakeeb A, Schmidt MC, Merchant NB, Parikh AA, Martin RC 2nd, Scoggins CR, Ahmad S, Kim HJ, Park J, Johnston F, Strouch MJ, Menze A, Rymer J, McClaine R, Strasberg SM, Talamonti MS, Staley CA, McMasters KM, Lowy AM, Byrd-Sellers J, Wood WC, Hawkins WG. Left-sided pancreatectomy: A multicenter comparison of laparoscopic and open approaches. Ann Surg 2008;248:438-446.

9. Fernandez-Cruz L, Cesar-Borges G, Lopez-Boado MA, Orduna D, Navarro S. Minimally invasive surgery of the pancreas in progress. Langenbecks Arch Surg 2005;390:342354.

10. Truty MJ, Sawyer MD, Que FG. Decreasing pancreatic leak after distal pancreatectomy: Saline-coupled radiofrequency ablation in a porcine model. J Gastrointest Surg 2007;11:9981007.

11. Hanly EJ, Mendoza-Sagaon M, Hardacre JM, Murata K, Bunton TE, Herreman-Suquet K, Poulose BK, Talamini MA. New tools for laparoscopic division of the pancreas: A comparative animal study. Surg Laparosc Endosc Percutan Tech 2004;14:53-60.

12. Fronza JS, Bentrem DJ, Baker MS, Talamonti MS, Ujiki MB. Laparoscopic distal pancreatectomy using radiofrequency energy. Am J Surg 2010;199:401-404.

13. Burdio F, Berjano EJ, Navarro A, Burdio JM, Grande L, Gonzalez A, Cruz I, Guemes A, Sousa R, Subira J, Castiella $\mathrm{T}$, Poves I, Lequerica JL. Research and development of a new RF-assisted device for bloodless rapid transection of the liver: computational modeling and in vivo experiments. Biomed Eng Online 2009;8:6.

14. Burdio F, Grande L, Berjano E, Martinez-Serrano M, Poves I, Burdio JM, Navarro A, Guemes A. A new single-instrument technique for parenchyma division and hemostasis in liver resection: A clinical feasibility study. Am J Surg 2010;200: 75-80.

15. Burdio F, Navarro A, Berjano E, Sousa R, Burdio JM, Guemes A, Subiro J, Gonzalez A, Cruz I, Castiella T, Tejero 
E, Lozano R, Grande L, de Gregorio MA. A radiofrequencyassisted device for bloodless rapid transection of the liver: A comparative study in a pig liver model. Eur J Surg Oncol 2008;34:599-605.

16. Navarro A, Burdio F, Berjano EJ, Guemes A, Sousa R, Rufas M, Subira J, Gonzalez A, Burdio JM, Castiella T, Tejero E, De Gregorio MA, Grande L, Lozano R. Laparoscopic bloodsaving liver resection using a new radiofrequency-assisted device: preliminary report of an in vivo study with pig liver. Surg Endosc 2008;22:1384-1391.

17. Kleeff J, Diener MK, Z'Graggen K, Hinz U, Wagner M, Bachmann J, Zehetner J, Muller MW, Friess H, Buchler MW. Distal pancreatectomy: Risk factors for surgical failure in 302 consecutive cases. Ann Surg 2007;245:573-582.

18. Reid-Lombardo KM, Farnell MB, Crippa S, Barnett $M$, Maupin G, Bassi C, Traverso LW. Pancreatic anastomotic leakage after pancreaticoduodenectomy in 1,507 patients: A report from the Pancreatic Anastomotic Leak Study Group. J Gastrointest Surg 2007;11:1451-1458.
19. Zhou W, Lv R, Wang X, Mou Y, Cai X, Herr I. Stapler vs suture closure of pancreatic remnant after distal pancreatectomy: A meta-analysis. Am J Surg 2010;200:529-536.

20. Ridolfini MP, Alfieri S, Gourgiotis S, Di Miceli D, Rotondi F, Quero G, Manghi R, Doglietto GB. Risk factors associated with pancreatic fistula after distal pancreatectomy, which technique of pancreatic stump closure is more beneficial? World J Gastroenterol 2007;13:5096-5100.

Address correspondence to: Dimitri Dorcaratto, $M D$ General Surgery Department Hospital del Mar

Paseo Maritimo 25-28

08003 Barcelona, Spain

E-mail: 97485@parcdesalutmar.cat 